\title{
Mobile Visual Communication
}

\author{
Photo Messages and Camera Phone Photography
}

\author{
MikKo ViLLI
}

\begin{abstract}
In this article I will elucidate the concept of photo messaging, and examine camera phones in the context of communication and photography. Camera functions are nowadays a popular add-on to the mobile (cellular) phone. Users can send photographs directly from the phone as photo messages. Findings suggest that the ubiquitous camera phone, and photo messaging, may substantially change the ways in which people use personal photography. The imaging capacity of mobile phones is becoming a potential part of perpetual visual contact. Thus taking and sending photographs on a camera phone represents a new resource for visual communication.
\end{abstract}

Key Words: photo message, MMS, photography, mobile communication, camera phone

\section{Introduction}

The aim of this article is to examine photo messages - both on a conceptual and practical level - and to review the meaning of camera phones in relation to communication and photography. The term "photo message" is not yet widely established; I use the term to refer to digital photographs taken with a camera phone (mobile phone with built-in digital camera) and sent to another mobile phone. A more general term is "MMS message"; MMS stands for Multimedia Messaging Service. However, MMS is, rather, the technological platform behind photo messaging. MMS-enabled mobile phones allow users to compose and send messages with one or more multimedia components (text, image, audio, video).

Other terms that have been utilized for photographs sent from a mobile phone are "picture message", "multimedia message", and "mobile visual message". The term picture message is not adequate as it is often used to refer to a drawing or a picture that can be sent from a phone using SMS (Short Message Service). For the purposes of my article I want to distinguish photo messages also from multimedia messages, as I am interested in photo messages particularly in relation to photographs; I do not include the study of text, audio, or video elements in my study, although photographs sent as MMS messages can be supplemented with other types of media content. In addition, a recent study (Ling \& Julsrud, 2005: 333) showed that the exchange of text and audio messages via MMS is rarely done, particularly since it often requires more time to develop and edit the messages. The main focus in MMS messaging is the exchange of photographs. Text messages (SMS messages), photo messages, multimedia messages, and picture messages unite under the term "mobile message". 
I approach the concept of photo message in two contexts: photo messages as a form of mobile communication, and photo messages in relation to photography. The time frame for my article is from the year 2000 onwards. Camera phones were introduced to the public in 2000, and MMS messaging in 2002. Thus all the empirical studies in camera phone photography and photo messaging that I refer to have been published during the last seven years, most of them in the past three years. Until now photo messaging has been studied mainly in computer and information sciences. An important goal, then, of this article is to introduce the concept of photo messaging to communication studies, as the phenomenon is relatively new to the field, and has not until date been greatly studied.

The article relies on secondary sources for its empirical material. Instead of presenting empirical data of my own, I will contemplate the findings of recent empirical studies concentrating on camera phone photography and photo messaging. Therefore this article does not offer new empirical results, but rather an overview of the phenomenon of photo messaging, and an effort to define a new concept in the context of communication studies. In addition to the empirical research publications (most of them published as conference papers), I utilize literature on both mobile communication and personal photography.

In the beginning of the article I will elucidate the concept of photo message, both in comparison to photographs, and as a new form of visual interpersonal communication. I will also deal briefly with the ways photo messaging has been researched until now. The latter part of the article consists of a review of the boundary-crossing uses of photo messaging in the context of personal photography, and most importantly, mobile phone communication.

\section{Significance of Camera Phones and Photo Messaging}

Built-in camera functions are now a popular and accepted add-on to the mobile phone. The camera phone is part of an overall trend towards non-voice functions in the world of mobile communication (Okabe, 2004). Many people opt just for the camera phone, instead of carrying with them both a mobile phone and digital camera. Already in the first half of 2003, camera phones outsold digital cameras worldwide. Within the next decade camera phones may become the predominant consumer imaging device. (Van House et al., 2004b: 2.)

Photo messaging can alter mobile phone communication from almost totally verbal (voice calls, SMS messages) into at least partly visual communication. ${ }^{1}$ When cameras are built into mobile phones, their imaging capacity becomes a potential part of perpetual visual contact (Koskinen, 2005b: 340-341). People are not limited to just photographing with their phones, as they can also send the photographs they take directly from the phone to somebody else - as photo messages. By using camera phones ordinary people can communicate visually over geographic distances, nearly in real time. ${ }^{2}$ Camera phones make possible an almost synchronous photographic contact with distant others. Just as the transition from exclusively land line based telephony to mobile telephony has made interpersonal communication more commonplace, increased access to photographs will make visual interaction more common (Ling et al., 2005: 96). The ability to share photographs from an ever-present mobile phone screen, or to send photographs from the mobile phone, means that image sharing will be much more commonplace (Kato et al., 2005: 305). In my view mobile interpersonal photographic communication constitutes a new genre in communication (see also Kindberg et al., 2005b). ${ }^{3}$ 
Photo messaging can also affect photography, in a sense that it causes photography to become more ubiquitous, transient, mundane, and interpersonal. Preliminary empirical findings suggest that ubiquitous digital image capture and sharing may substantially change the ways people use personal photography - potentially it may be the most radical development in photography since the film camera made personal photography possible (Van House et al., 2005: 1853). The essential difference between an ordinary camera and a camera phone is that the camera phone represents not only an ever-present image capture device but also an ever-present image sharing and transmission device (Kato et al., 2005: 305).

\section{Concept of Photo Message}

The concept of photo message is not yet very clearly established, and there exists a slightly confusing terminological division: terms in use are photo message, picture message, multimedia message, MMS message, and mobile visual message. MMS messaging is a standard based on text messaging (SMS) where people can send a message to one or several recipients. MMS messages can be sent to other MMS compatible phones or directly to email addresses. (Jacucci et al., 2005.) As mentioned earlier, for the purposes of this article I want to clearly differentiate photo messages from MMS messages in general, as I focus on photo messaging solely in the context of photography, not audio or video communication.

\section{Photo Messages and Photography}

An indispensable way to define the concept of photo message is to examine it in conjunction with photographs. When does a photograph turn into a photo message? In my view, a photograph can be classified as a photo message at the moment when it is sent from the mobile phone; until that moment it is just a photograph taken with a mobile phone. Therefore a photo message is by definition a photograph used in a certain way. However, photo messages are not (at least not yet) a certain form of photograph or genre of photography (cf. family photograph, journalistic photograph, passport photograph etc.). By looking at a photo message "as a photograph" we cannot specify anything that would specifically make it a photo message.

Also photographs sent as e-mail attachments from mobile phones can be classified as photo messages. In contrast, although we can send a photograph from a computer via e-mail or put a printed photograph in an envelope and send it by ordinary mail, these are rarely referred to as photo messages. Thus, the technical requirements for a photo message are a mobile phone equipped with a camera and an MMS or e-mail application.

Many of the photographs taken with a mobile phone are never sent to another phone, but are merely looked at on the screen of the phone, transferred to a computer, or delivered to a moblog ${ }^{4}$ on the web. It is correct to say that these images are just "photographs" that are never transformed into photo messages. At the same time, we have to remember that all photo messages are also photographs, so it is quite impossible to construct a strong dichotomy between photo messages and photographs. The distinction between photographs and photo messages is artificial, but in this article I call a photograph a photo message when it is sent from a mobile phone to some other person(s). People thus take photographs on their mobile phones and use some of them as photo messages. This distinction is necessary when trying to study photo messages in the context of communication. 


\section{Photo Messages and Communication}

In the context of this article, particularly interesting is the communicational use of photographs, especially when photographs are used as messages. Of course, all photographs are also messages: they communicate something to somebody, they are compositions of information (Wiio, 1989) and objects of communication. A photograph is an utterance of some sort, in that it carries, or is, a message (Sekula, 1982: 85). But in some way photo messages must be more like "messages" than ordinary photographs, because we call them photo messages, not just "mobile photographs" or "camera phone photographs".

In my view, the justification for calling photo messages "messages" is that they are sent, not shown. Photo messages are not viewed on the screen of the photographer's mobile phone but rather on the screen of the recipient's phone. Photo messages cross geographical distances when traveling through cyberspace..$^{5}$ Although immaterial and digital at every stage ${ }^{6}$, photo messages appear to be concrete messages that move from one place to another (for instance from a mobile phone in Australia to a phone in Finland). Essential to a "message" is that it is intended to be communicated from one person to another; a transfer of communication substance occurs. A photograph is also an object of communication, but it is not necessarily meant to be transferred, or communicated directly to somebody else.

Photo messages are a form of interpersonal communication. Interpersonal communication is commonly designated as happening between two communicators (Knapp et al., 2002: 9). Photo messages are mainly, though not always, one-to-one communication, whereas photographs are generally one-to-many. The photographs in an album or on the wall are meant for a more general audience consisting of friends, relatives, and so on. The camera phone is strongly associated with an individual viewpoint, while the traditional camera tends to take on more of the role of a third party (Okabe, 2004).

Koskinen and Kurvinen (2005: 5) state that MMS is not just a technology for interaction; interaction is an essential phenomenon to be studied if we want to understand mobile multimedia messaging. Kindberg et al. (2004) add that camera phones are not simply extensions of already existing devices (such as mobile phones or digital cameras), but rather enablers of new forms of interaction. To represent camera phones primarily as "capture and show" rather than "capture and send" devices would be to misrepresent the range of activities they support. The ability to bring remote friends and family into a visual experience, or the achievement of tasks with remote people through the use of images, are supported by the particular combination of camera with direct sending capabilities. (ibid: 12.)

\section{Previous Research}

Due to the novelty of camera phones and photo messaging, research in the field is in its early stages. ${ }^{7}$ However, it can be expected that the amount of research will increase as the number of mobile phones with cameras grows, and also as the sending of photo messages becomes (technically and economically) less demanding for the users.

MMS messaging was introduced to the public in 2002. Already before that the first research projects in mobile photography and photo messaging were carried out with prototypes that were not actual MMS phones. In a study project Maypole in 1997-1998 a prototype device was developed consisting of a camera, an interface module, and a laptop in a rucksack (Mäkelä et al., 2000). In the study Mobile Image (Koskinen et al., 2002) in 
1999-2000 the users could send "photo messages" using a mobile phone and a digital camera connected with an infrared link. Good background for photo messaging is provided also by literature on text messaging (several articles in edited books such as Katz \& Aakhus, 2002a; Harper et al., 2005; Nyíri, 2005; see also Grinter \& Eldridge, 2003).

As the possibility to send photo messages is a very new addition to mobile phones, most of the studies done in the area can be called "design studies" - studies of technology that does not yet exist on the marketplace. Design studies offer a unique opportunity to combine technology, design, and behavioral issues to inform technology and service development. (Koskinen, 2005a.) Research is thus not often made in order to understand the phenomenon but to create better products and services. Still, the studies (such as Mäkelä et al., 2000; Koskinen et al., 2002; Sarvas et al., 2004; Van House et al., 2005; Davis et al., 2005) provide a lot of insights into the ways users conceive the possibility to communicate photographically over geographic distances.

Most of the design studies have been made with prototype configurations or prototype systems (both hardware and software). In most cases people have got access to such technology that they would not have had without the study. There exist only few studies that focus on "real users", people who use a product or application that has already been introduced to the market and is in commercial use.

However, in contrast to design studies, another line of research has begun to explore actual users and technology through interviews, by discussing messages people have taken, and sometimes, by observing the users. When contrasted to a traditional design study with a mock-up virtual prototype of an interface or with mere usability studies, this study pays serious attention to sociability as a driving force in mobile multimedia. (Koskinen, 2005a.) However, the samples of users in the studies done with existing technology have been quite small. Mobile multimedia has also been too minor a phenomenon to be yet studied purely from statistics of large segments of camera phone users. The line of study with real users (e.g. Kurvinen, 2003; Kindberg et al., 2004; Ling \& Julsrud, 2005; Ling et al., 2005; Oksman, 2005; Scifo, 2005) has, naturally, been carried out only in the past few years, after the introduction of MMS services. The area clearly needs more theoretical, academic research in addition to the design studies that often have ties with marketing research.

\section{Mobile Phone Photography}

In the following section I will review photo messaging and camera phone photography in relation to personal photography. I will not focus on photography as mass communication, or photojournalism, because photography in the context of mobile communication belongs almost totally to the realm of personal photography; professional or journalistic photography is rarely practiced with camera phones. ${ }^{8}$ I will elucidate the different ways camera phones and photo messages affect personal photography, namely causing photography to become a more mundane activity, and directing photography more towards acting in the "present tense".

The different uses of "traditional" personal photographs (taken with an ordinary camera) have been classified as follows: constructing personal and group memory, creating and maintaining social relationships, and self-expression and self-presentation (Van House et al., 2004b), as well as documentation, memory support, and definition of cultural membership (Chalfen, 1987). A good way to specify personal photography is to tie it to the home mode of communication formulated by Chalfen (ibid). Snapshots 
and home movies are forms of home mode communication, which is described as a pattern of interpersonal and small group communication. Photographers usually know the people in their pictures, and viewers usually know the photographer, and, most of the time either know or can identify the subjects of the pictures.

Family photography is a focal part of personal photography. In family photography the people in the pictures are related to each other, either by kin or friendship. Family photography sustains the notion of the family as a corporate entity - parties, picnics, vacations etc. are occasions when family photography takes place. (Hirsch, 1981.) It would be highly unconventional to take photographs of strangers for a family album (Frohlich, 2004: 37-38).

Besides camera phone photography, also photo messaging as photography belongs to the context of personal photography, because photo messages do not circulate in the public, but are strictly private, interpersonal communication, (mostly) between two mobile phones, comparable to a phone call or text message. Photo messaging is thus not a part of mass communication. ${ }^{9}$

\section{Uses of Camera Phones}

Camera phones are often used for more functional images - photographs in place of writing, copying, or scanning (Van House et al., 2005: 1855-1856); support of the accomplishment of a particular task (Kindberg et al., 2005a: 1546); but also for affective (sentimental, emotional) reasons (Kindberg et al., 2005b: 44). In addition, personal archiving, intimate sharing, and peer-to-peer news and sharing (Okabe, 2004) have been noted as patterns of camera phone use.

Camera phones are much more than a "poor relation" to digital and conventional cameras, despite some concerns about image quality (Spasojevic, 2005: 2). Their ubiquitous presence invites a new kind of personal awareness, a persistent alertness to the visually newsworthy (Okabe \& Ito, 2003). Ready access to imaging encourages people to see the world "photographically" - as images. With a camera always available (in the mobile phone) and with the addition of easy viewing and sharing of photographs, phototaking becomes for many a frequent, even daily, activity. People have found new ways of using images for enduring social uses (such as communicating with loved ones) as well as activities for which photos were not previously used (e.g., reminders). (Van House et al., 2005: 1856.) The camera phone works also well as a "photographic memory": a photographic archive of memories, a mobile archive always within easy reach, something to look at again and again (Scifo, 2005: 365). Because of the camera phone's ability to capture and view images anywhere, the subjects often use their camera phones as personal flipbooks of images (Kindberg et al., 2005b).

Compared to the traditional camera, which is mainly used for special excursions and events - noteworthy moments bracketed off from the mundane - camera phones capture moments more fleeting and unexpected. "The mundane is elevated to a photographic object" (Okabe \& Ito, 2003) - a cake that looked good at a café, or a sudden encounter with a cute child or pet (Ito, 2004). Camera phones change the definition of photoworthy, from what is special and enduring to what is often transitory and ordinary (Van House et al., 2005: 1854).

Personal photography has not earlier included much this type of mundane photography. Chalfen (1987: 94) writes in his influential book "Snapshot Versions of Life" (dating to times before digital photography and camera phone photography) that we seldom 
if ever find snapshot images of people taking showers, brushing teeth, combing hair, shaving, using the toilet, people preparing breakfast or dinner, washing the dishes, vacuuming, dusting, polishing furniture or silverware, or otherwise cleaning the house. Nor do we see people reading newspapers or books, writing a letter, using the telephone, listening to a radio or stereo, watching television, playing cards or board games.

The traits of camera phone photography relate to all photographs taken with mobile phones, not just photo messages. However, it can be expected that photo messages are as mundane and fleeting as any other camera phone photograph (or even more so). Early research from 1999-2000 by Koskinen et al. (2002) already showed that sending mobile images was used mainly for communicating experiences and immediate feelings.

\section{Power of Now}

In addition to camera phones being used to photograph the more mundane objects and events, camera phones can also affect personal photography in relation to time. In the mobile phone the photograph is ready to be sent immediately. Contrary to the "what is" of photo messages, ordinary photographs are normally used to show "what was" - they work in the past tense. Photo messages often work not as memories or documents of the past but as transient and interpersonal messages - they happen here and now. In this sense a photo message is close to the present tense, describing something that is happening (almost) at the moment. A photograph is a document of me having been there, a photo message is a document of me being here - a way to share the moment visually with someone. The phone enables immediate communication, and the same immediacy characterizes mobile photographic communication.

MMS messages (i.e. photo messages) ${ }^{10}$ can thereby act as a contemporary form of testimony and authentication of one's presence in a certain physical space. By sending an MMS message, users aim to narrate what they are doing and experiencing right then, right there. (Scifo, 2005: 368-369.) The sense of real time capturing and sharing ("Power of Now") seems to be important to the senders (Van House et al., 2004a). Camera phones are used to communicate with people who are not present to share an experience when a picture is taken (Kindberg et al., 2005a).

Photographs have been generally treated as documents of the past. According to Barthes (1991: 44) a photograph does not establish a consciousness of the being-there of the thing, but an awareness of its having-been-there, for in every photograph there is the always stupefying evidence of this is how it was. However, in a photo message the "past" of the photograph is not always temporally much apart from the present.

To conclude: the photo message is a similar document of something having happened, as with any photograph. Still, there seems to be a difference in the content of mobile images. Photo messages are ephemeral, transient, and interpersonal by nature, and they are usually intended for some specific recipient, not for the general public or yet unborn grandchildren. They are meant to be looked at now, not in the near or distant future.

\section{Photo Messages in the Context of Mobile Phone Communication}

Mobile phone communication is determined by two characteristics: it is interpersonal and remote. According to Weilenmann (2003: 23-24) mobility does not necessarily mean that people move around. They can be sitting down at a table, using mobile technology to interact in some way with other people. 
Katz and Aakhus (2002b: 312) note that much of communication theory draws from face-to-face interpersonal communication and mass media modes. Beginning in the 1970s, a third and increasingly visible branch of theory was evolving, centered on understanding human-computer interaction. According to them it is now appropriate to speak of a fourth form of communication mediated through personal technologies. Rule (2002: 242) coins the term particularizing communications, which, unlike mass communication, are not standardized, one-way transmissions, but exchanges of highly specific, interactive data between individuals and organizations. ${ }^{11}$

According to Ling (2004) the niche of mobile telephone is in the realm of small groups for microcoordination of activities. Microcoordination is the nuanced management of social interaction. Koskinen (2003: 646) states that mobile technology is Janusfaced: it supports both expressive and instrumental activities, as well as ordinary interactions.

The term telematisation can be used to describe the fact that everyday life is penetrated by long distance communication media (Höflich \& Gebhardt, 2005: 9). With a mobile phone a person has a running sense of the other's location and situation. Thus, there exists a type of remote presence. (Ling, 2004: 192.) Mobile communication also extends a condition that Gergen (2002: 227) calls absent presence. One is physically present but is absorbed by a technologically mediated world elsewhere.

Mobile phones help to re-establish bonds over space and time in contexts where core features of domestic life are threatened and have to be reasserted, such as coordination of home life with workplace situations, or motherly and fatherly devotion towards children (Licoppe \& Heurtin, 2002: 95). With mobile technology one's communication time is increasingly spent in the presence of "those who matter" (Gergen, 2002: 238). Ito and Okabe (2005) present the term ambient virtual co-presence to describe the way people use mobile phones (especially their messaging capabilities) to maintain ongoing background awareness of others. Habuchi (2005) calls a sphere of intimacy that is free of geographical and temporal restraints a telecocoon. People who form telecocoons can generally be characterized as being constantly attentive to their group of friends.

Mobile phone use and personal photography share the aspect that they are communication of or to those "who matter". Thus in the context of photo messaging the most important feature of mobile phone communication is intimate interpersonal communication.

The conventions of mobile phone culture and telephone use can be thought to determine the use of photographs taken with a camera phone. The mobile phone is an interactional, two-way communication device. People are used to sending and receiving messages with it (until now only verbal messages). In comparison with the traditional camera, most of the photographs taken with a camera phone are short-lived and ephemeral images (Okabe, 2004). Photography cannot become as "live" as a phone call, but photo messages might narrow the gap significantly. Photo messages can be considered the extreme case of immediacy in photography (immediacy in reference to the speed of communication, not the illusion of the omission of the medium; see Bolter and Grusin, 2000).

\section{Photo Messages as Connection}

It has to be noted that photographs have been used for purposes of private visual communication since the invention of the photographic process around 1839 (Chalfen, 1987: 71). Perhaps the most important social role of personal photography is building and maintaining social relationships by sharing photographs. Among other things, printed 
photos provide support for face-to-face interactions with friends and family. Gathered around loose prints or bound albums, people tell stories and reminisce about shared experiences. (Van House et al., 2004b: 9.) People experience as much joy from the feedback and subsequent conversation arising from the photos they have sent, as they do from sending or receiving the photos themselves. This sharing is seen as a way of recreating the past and reliving the experience with others. (Frohlich et al., 2002.)

In addition to face-to-face sharing, photographs can also be shared by direct phoneto-phone transfer, either over infrared, Bluetooth, MMS, or e-mail connection. In the cases of MMS and e-mail, however, discussion over the photographs is less convenient as the people do not share the same physical location. ${ }^{12}$ But, with the aid of photo messages people can have a discussion with photographs, not just a discussion over photographs. This "pictorial dialogue" as a form of personal interaction has been found to be an important aspect of photo message use (Koskinen et al., 2002).

MMS can make possible one mode of photo-sharing, namely the distant but synchronous exchange of photographs by messaging: the photographs form a simple connection, "like a kiss or a hug" (Van House et al., 2004b: 7). What is essential is that camera phones make photographic connection easy with distant loved ones and friends. Communicating through images is closely and almost exclusively linked to one's network of strong relationships. (Scifo, 2005: 367-368.) Research subjects have explained that they send pictures of daily life to family, for information, but even more, for connection. While any kind of camera could take these pictures, they specifically use the camera phone to send casual, spontaneous photographs of everyday life and sights (e.g., a picture of a tree in full bloom sent to family in a location where it is snowing). (Van House et al., 2005: 1855.)

Taking and sending photographs on a camera phone represents thus a new communication resource that can be invested in one's peer group and in one's relationships (Scifo, 2005: 365). Since people can respond immediately to MMS messages (commenting a picture with a picture), mobile phones provide an interactive social context for photography (Koskinen, 2005b: 340-341; see also Koskinen et al., 2002). Camera phones make ubiquitous visual access to others possible (Okabe, 2004). The combination of camera and direct sending capabilities lets people use images to bring remote others into an experience (Kindberg et al., 2005b: 48). The camera phone seems to respond to a need on the part of users to appropriate their social spaces visually. Remote, simultaneous visual communication is becoming a way to take hold of the experience and the world of others. (Scifo, 2005:370-373.) Drawing someone visually into an experience in real time despite being separated by distance represents a compelling way to stay close (and is an example of strong telepresence) (Kindberg et al., 2005b: 46).

Japanese pilot studies of photo sharing among young couples and peer groups show that couples and close groups of female friends have the highest volume of image transmission; among male friends and less intimate relations, images are rarely transmitted, although they may be shared from the handset when people are physically copresent. Visual sharing is most prevalent among "full time intimate communities". (Kato et al., 2005: 306.) Full time intimate communities consist of frequent contacts with a select few; it is a roundthe-clock set of relationships with an exclusive group of friends (Matsuda, 2005: 133).

Thus a telecocoon can be effectively maintained by using photographs sent as photo messages. The photographs can be of the mundane things in life, because they do not need to be informative or esthetically sophisticated, but instead they function as mediators of the daily routines of "my life" and help to visually take hold of the experience and the world of others not present. 
In a sense, photo messages can be compared to postcards (see Lehtonen et al., 2003). Östman and Laakso (1999: 17-18) state that people do not send postcards in order to say anything special or articulate, but to announce that they are alive, and that they give their thoughts to the recipient. According to Ling et al. (2005: 92) MMS sits at the nexus between the postcard and the family album. It has the ability to capture travel experiences just as with traditional photography. However, it has the additional feature of allowing images to be sent to others like postcards. Postcards and photo messages are both a form of visual telepresence and interpersonal communication.

It can be concluded that photo messaging is strongly connected to the ritual view of communication. According to the definition by James Carey (1989), the ritual view of communication is linked to terms such as "sharing", "participation" "association", and "fellowship". It can be distinguished from the transmission view of communication where communication is perceived as one-way transfer of messages, and defined by terms such as "imparting", "sending", "transmitting", or "giving information to others". Even before the invention of mobile phone photography, personal photographs (e.g. family photographs) have mainly been used in the context of the ritual view of communication. The possibility provided by a camera embedded in an interpersonal communication device (mobile phone) now reinforces photography as ritual communication.

\section{Obstacles of Photo Messaging}

In spite of the above-mentioned possibilities and reasons for mobile sharing of camera phone photographs, face-to-face sharing (i.e., showing photographs on the screen of the mobile phone) seems, at least for now, to be the more popular way of sharing camera phone photographs. In a study (Kindberg et al., 2005a) including 34 camera phone users in the U.S. and U.K., the subjects used face-to-face sharing for the majority of the images, and remote sending (largely MMS) for only little more than a third of the photographs. Received images comprised less than eight percent of the images on the subjects' phones, the great majority (92\%) being their own captured photographs.

In choosing the sharing mechanism, of significance were the general barriers to sending photo messages, including the lack of a "critical mass" of people to exchange images with, expense, complexity, and poor image quality (ibid: 1546). Other studies (Scifo, 2005) have also listed various technical problems in sharing photographs from the camera phone, such as the problem of there being many ways (MMS, e-mail, http upload, Bluetooth, and infrared) to share photos from the camera phone, but none of which are easy and consistent.

In addition to the mainly technical reasons, social reasons can also limit people's sending of photo messages. Apparently, in comparison with sending text messages, sending photos might be perceived as "intrusive", and "narcissistic". Users prefer to share images by showing pictures on a mobile phone screen; most of the photos that people take are not intended to be sent to others. (Okabe, 2004.) This underlines the sociality of viewing photographs together. Additionally, if the image or its meaning is insufficiently clear, then showing and interpreting it in person may be the only option (Kindberg et al., 2005a: 1548).

Maybe then the inherent value of camera phones does not lie in the sending of images (Kindberg et al. 2004, 1). The MMS-enabled camera phone is still mostly closer to a "digital wallet" - i.e. a miniature photo album or a digital photo archive - than a multimedia communication device (Oksman, 2005: 359). People might be satisfied with 
text messages and do not see the need to send photographic messages. Jenson (2005: 315 ) argues that MMS clearly will not replace SMS usage, and in order to succeed, it needs to find a new, as yet undiscovered, social need that will enthusiastically embrace photo messaging.

\section{Conclusion}

In this article I have reviewed the emerging field of mobile visual communication. The focus has been on photo messaging and camera phone photography. Photo messages are digital photographs taken with a camera phone and sent to another mobile phone. Photo messages can be categorized as a form of visual interpersonal communication.

A salient point is the affiliation between photo messages and mobile phones. The telephone is a two-way communication device, in which interpersonal communication and real time messaging are emphasized. Photographs sent as photo messages enable visual telepresence almost in real time. Photo messages help to maintain a visual telecocoon of an intimate community, regardless of the geographical distance between the community members. Camera phones can affect also photography by making it more transient, mundane, and interpersonal.

Photo messaging can alter mobile communication from almost totally verbal into at least partly visual communication. The motivation behind sending photo messages might not be as much to transmit information but rather to share presence by visual means. An interesting aspect is the idea of pictorial conversation - conversation or dialogue with photographs, where people use their mobile phones to send photo messages back and forth to each other, commenting pictorially on the photographs they receive (see Koskinen et al., 2002). Deeper research in the context of interpersonal pictorial conversation is the subject of my future study. I am especially interested in developing the hypothesis of photo messaging forming a new genre in communication - mobile interpersonal photographic communication.

In all, the camera phone is located on the border between new and old communication behavior, and the device is still much compared to the camera (Oksman, 2005: 360). Camera phone photographs are often highly personal views of everyday life that are archived on the small screen - "The camera phone is my eye" (Okabe, 2004). From this, it follows that since camera phone photographs are often taken for purely personal use, there is not necessarily any great need for sharing them. This is a significant difference in contrast to the other communicational uses of mobile phones, such as voice calls and text messaging.

Users might not want to act in a more communicative way with photographs, even though they take the photographs with a mobile phone, which is very much a communication device, unlike the traditional camera apparatus. ${ }^{13}$

\section{Notes}

1. Visual contact with distant others can be achieved from the mobile phone also by using video calls, email or web applications.

2. The instantaneous or even synchronous, remote visual contact has been made possible for common people also by introducing instant messaging (IM) systems.

3. I will not develop the idea of the new genre further in this article, but it will be the focus of my research in the future. 
4. A moblog is like a weblog, but consists mainly of periodic posts of (user-taken) mobile phone photographs. The main idea of moblogging is that unlike with regular digital cameras, there is no intermediate transfer step between capturing the photo and publishing it (Jacucci et al, 2005).

5. Sending photographs from one mobile phone to another via a Bluetooth connection is a borderline case, as the transfer of a photograph takes place, but the recipient can be only about 10 meters away, due to the limitations of Bluetooth technology.

6. According to my definition, photo messages appear only on the screen of a mobile phone. They do not exist as separate pictorial artifacts.

7. A lot of research has been made by mobile phone and telecommunications companies, but their studies are mostly confidential.

8. However, it should be noted that newspapers have already used photographs taken with camera phones (e.g. the notorious photographs taken in the Abu Ghraib prison in Iraq). In November 2004 a daily Amsterdam newspaper, De Telegraaf, became news on its own when it published a picture taken with a mobile phone of Dutch filmmaker Theo van Gogh's body moments after he was killed. "Mobile picture power in your pocket”. BBC News Saturday, 13 November, 2004. http://news.bbc.co.uk/2/hi/ technology/3991775.stm

9. Photographs can be sent from a mobile phone to a moblog for the masses to see it, but I do not include moblogs to be a part of photo messaging.

10. The cited sources widely use the term "MMS message." However, which of the different media types the MMS messages contain is rarely identified. I understand most of the MMS messages to be photo messages, as they primarily contain photographs.

11. Mobile phones can also function as receivers of mass communications, for instance when SMS news messages or mobile TV broadcasts are delivered to the phone.

12. Various studies (e.g. Sarvas et al., 2004; Davis et al., 2005) have focused on developing systems to enable remote sharing of camera phone photographs.

13. It is not wise, however, to base one's argument too strongly on the differences between a camera and a phone, as in the near future we might photograph and communicate mainly with a general media device. The camera as a "pure" camera might be of interest only to artists and professional photographers.

\section{References}

Barthes, R. (1991) Image, Music, Text. Essays selected and translated by Stephen Heath (orig. 1977). New York: The Noonday Press.

Bolter, J. \& Grusin, R. (2000) Remediation. Understanding New Media. Cambridge, MA: The MIT Press.

Carey, J. (1989) Communication as Culture: Essays on Media and Society. Winchester, MA: Unwin Hyman.

Chalfen, R. (1987) Snapshot Versions of Life. Bowling Green, Ohio: Bowling Green State University Popular Press.

Davis, M, Van House, N., Towle, J., King, S., Ahern, S., Burgener, C., Perkel, D., Finn, M., Viswanathan, V. \& Rothenberg, M. (2005) 'MMM2: Mobile Media Metadata for Media Sharing', in Extended Abstracts of the Conference on Human Factors in Computing Systems (CHI 2005) in Portland, Oregon, April 2-7, 2005. New York: ACM Press.

Frohlich, D., Kuchinsky, A., Pering, C., Don, A. \& Ariss, S. (2002) 'Requirements for Photoware', in ACM Conference on Computer Supported Cooperative Work, November 16-20, 2002. New York: ACM Press.

Frohlich, D. (2004) Audiophotography. Bringing Photos to Life with Sounds. Dordrecht: Kluwer Academic Publishers.

Gergen, K. (2002) 'The Challenge of Absent Presence', in Katz, J.E. \& Aakhus, M. (eds.) Perpetual Contact. Mobile Communication, Private Talk, Public Performance. Cambridge: Cambridge University Press.

Grinter, R. \& Eldridge, M. (2003) Wan2tlk?: Everyday Text Messaging. CHI 2003, April 5-10, 2003 , Ft. Lauderdale, Florida, USA. New York: ACM Press.

Habuchi, I. (2005) 'Accelerating Reflexivity', in Ito, M., Okabe, D. \& Matsuda, M. (eds.) Personal, Portable, Pedestrian. Mobile Phones in Japanese Life. Cambridge, MA: The MIT Press.

Harper, R., Palen, L. \& Taylor, A. (eds.) (2005) The Inside Text. Social, Cultural and Design Perspectives on SMS. Dordrecht: Springer.

Hirsch, J. (1981) Family Photographs. Content, Meaning, and Effect. Oxford University Press: New York. 
Höflich, J.R. \& Gebhardt, J. (2005) 'Changing Cultures of Written Communication: Letter - E-mail SMS', in Harper, R., Palen, L. \& Taylor, A. (eds.) The Inside Text. Social, Cultural and Design Perspectives on SMS. Dordrecht: Springer.

Ito, M. (2004) Personal Portable Pedestrian: Lessons from Japanese Mobile Phone Use. Paper presented at Mobile Communication and Social Change, the 2004 International Conference on Mobile Communication in Seoul, Korea, October 18-19, 2004. http://www. itofisher.com/mito/archives/ ito.ppp.pdf, acquired May 12, 2005.

Ito, M. \& Okabe, D. (2005) 'Technosocial Situations: Emergent Structuring of Mobile E-mail Use', in Ito, M., Okabe, D. \& Matsuda, M. (eds.) Personal, Portable, Pedestrian. Mobile Phones in Japanese Life. Cambridge, MA: The MIT Press.

Jacucci, G., Oulasvirta, A., Salovaara, A. \& Sarvas, R. (2005) 'Supporting the Shared Experience of Spectators through Mobile Group Media', Proceedings of the 2005 international ACM SIGGROUP conference on Supporting group work GROUP '05, November 6-9, 2005, Sanibel Island, Florida, USA. ACM Press: New York.

Jenson, S. (2005) 'Default Thinking: Why Consumer Products Fail', in Harper, R., Palen, L. \& Taylor, A. (eds.)The Inside Text. Social, Cultural and Design Perspectives on SMS. Dordrecht: Springer.

Kato, F., Okabe, D., Ito, M. \& Uemoto, R. (2005) 'Uses and Possibilities of the Keitai Camera', in Ito, M., Okabe, D. \& Matsuda, M. (eds.) Personal, Portable, Pedestrian. Mobile Phones in Japanese Life. Cambridge, MA: The MIT Press.

Katz, J.E. \& Aakhus, M. (eds.) (2002a) Perpetual Contact. Mobile Communication, Private Talk, Public Performance. Cambridge University Press, Cambridge, UK.

Katz, J.E. \& Aakhus, M. (2002b) 'Introduction: Framing the Issues', in Katz, J.E. \& Aakhus, M. (eds.) Perpetual Contact. Mobile Communication, Private Talk, Public Performance. Cambridge: Cambridge University Press.

Kindberg, T., Spasojevic, M., Fleck, R. \& Sellen, A. (2004) How and Why People Use Camera Phones. Consumer Applications and Systems Laboratory. H\&P Laboratories Bristol, HPL-2004-216, November 26, 2004.

Kindberg, T., Spasojevic, M., Fleck, R. \& Sellen, A. (2005a) 'I saw This and Thought of You: Some Social Uses of Camera Phones', in Extended Abstracts of the Conference on Human Factors in Computing Systems (CHI 2005) in Portland, Oregon, April 2-7, 2005. New York: ACM Press.

Kindberg, T., Spasojevic, M., Fleck, R. \& Sellen, A. (2005b) 'The Ubiquitous Camera: An In-Depth Study of Camera Phone Use', IEEE Pervasive Computing, Apr-Jun., 2005.

Knapp, M., Daly, J., Fudge Albada, K. \& Miller G. (2002) 'Background and Current Trends in the Study of Interpersonal Communication', in Knapp, M. \& Daly J. (eds.) Handbook of Interpersonal Communication. $3^{\text {rd }}$ edition. Thousand Oaks: Sage.

Koskinen, I., Kurvinen, E. \& Lehtonen, T-K. (2002) Mobile Image. Helsinki: IT Press.

Koskinen, I. (2003) 'User-Generated Content in Mobile Multimedia: Empirical Evidence from User Studies', Proceedings of International Conference of Multimedia and Expo ICME 2003. Baltimore: IEEE Publication.

Koskinen, I. (2005a) Pervasive Image Capture and Sharing: Methodological Remarks. Paper presented at Ubicomp '05 The Seventh International Conference on Ubiquitous Computing. September 11-14, 2005, Tokyo, Japan. http://www.spasojevic.org/pics/PICS/Ubicomp05WS2Koskinen.pdf, acquired September 16, 2005.

Koskinen, I. (2005b) 'Seeing with Mobile Images: Towards Perpetual Visual Contact', in Nyíri, K. (ed.) A Sense of Place: The Global and the Local in Mobile Communication. Vienna: Passagen Verlag.

Koskinen, I., \& Kurvinen, E. (2005) 'Mobile Multimedia and Users: On the Domestication of Mobile Multimedia', Telektronikk 3/4.2005. http://www.telenor. com/telektronikk/volumes/front.php, acquired January 27, 2006.

Kurvinen, E. (2003) Only When Miss Universe Snatches Me. Teasing in MMS Messaging, in Proceeding of Designing Pleasurable Products and Interfaces DPPI'03, Pittsburgh, Pennsylvania, 2003.

Lehtonen, T.-K., Koskinen, I. and Kurvinen, E. (2003) Mobile Digital Pictures - the Future of the Postcard. 9th Interdisciplinary Conference on Research in Consumption. Dept. of Design History/Material Culture, University of Applied Arts, Vienna, Austria, June 27-29, 2003.

Licoppe, C. \& Heurtin, J-P. (2002) 'France: Preserving Image', in Katz, J.E. \& Aakhus, M. (eds.) Perpetual Contact. Mobile Communication, Private Talk, Public Performance. Cambridge: Cambridge University Press.

Ling, R. (2004) The Mobile Connection. The Cell Phone's Impact on Society. San Francisco: Morgan Kaufmann Publishers. 
Ling, R. \& Julsrud, T. (2005) 'Grounded Genres in Multimedia Messaging', in Nyíri, K. (ed.) A Sense of Place: The Global and the Local in Mobile Communication. Vienna: Passagen Verlag.

Ling, R. Julsrud. T. \& Yttri, B. (2005) 'Nascent Communication Genres within SMS and MMS', in Harper, R., Palen, L. \& Taylor, A. (eds.) The Inside Text. Social, Cultural and Design Perspectives on SMS. Dordrecht: Springer.

Matsuda, M. (2005) 'Mobile Communication and Selective Sociality', in Ito, M., Okabe, D. \& Matsuda, M. (eds.) Personal, Portable, Pedestrian. Mobile Phones in Japanese Life. Cambridge: The MIT Press.

Mäkelä, A., Giller, V, Tscheligi, M. \& Sefelin, R. (2000). 'Joking, Storytelling, Artsharing, Expressing Affection: a Field Trial of How Children and their Social Network Communicate with Digital Images in Leisure time', Proceedings of the SIGCHI conference on Human Factors in Computing Systems. Hague, The Netherlands.

Nyíri, K. (ed.) (2005) A Sense of Place: The Global and the Local in Mobile Communication. Vienna: Passagen Verlag.

Okabe, D. \& Ito M. (2003) 'Camera Phones Changing the Definition of Picture-Worthy', Japan Media Review 2003, http://www.ojr.org/japan/wireless/1062208524. php, acquired May 17, 2005.

Okabe D. (2004) Emergent Social Practices, Situations and Relations through Everyday Camera Phone Use. Paper presented at Mobile Communication and Social Change, the 2004 International Conference on Mobile Communication in Seoul, Korea, October 18-19, 2004. http:// www.itofisher.com/mito/archives/okabe_seoul.pdf, acquired May 12, 2005.

Oksman, V. (2005) 'MMS and Its 'Early Adopters' in Finland', in Nyíri, K. (ed.) A Sense of Place: The Global and the Local in Mobile Communication. Vienna: Passagen Verlag.

Rule, J.B. (2002) 'From Mass Society to Perpetual Contact: Models of Communication Technologies in Social Context', in Katz, J.E. \& Aakhus, M. (eds.) Perpetual Contact. Mobile Communication, Private Talk, Public Performance. Cambridge: Cambridge University Press.

Sarvas, R., Viikari, M., Pesonen, J., \& Nevanlinna, H. (2004) 'MobShare: Controlled and Immediate Sharing of Mobile Images', Proceedings of the 12th annual ACM international conference on Multimedia in New York, October 10-16, 2004. New York: ACM Press.

Scifo, B. (2005) 'The Domestication of Camera-Phone and MMS Communication: The Early Experiences of Young Italians', in Nyíri, K. (ed.) A Sense of Place: The Global and the Local in Mobile Communication. Vienna: Passagen Verlag.

Sekula, A. (1982) 'On the Invention of Photographic Meaning', in Burgin, V. (ed.) Thinking of Photography. London: Macmillan.

Spasojevic, M. (2005) Camera Phone Use and its Implications. Paper presented at Ubicomp '05 The Seventh International Conference on Ubiquitous Computing. September 11-14, 2005, Tokyo, Japan. http://www.spasojevic.org/pics/PICS/Ubicomp 05WS2Koskinen.pdf, acquired September 16, 2005.

Van House, N., Davis, M., Takhteyev, Y., Good, N., Wilhelm, A. \& Finn, M. (2004a) From 'What?' to 'Why?': The Social Uses of Personal Photos. School of Information Management and Systems (SIMS), University of California, Berkeley, Working Papers. http://www.sims.berkeley.edu/ $\sim$ vanhouse/photo_project/pubs/vanhouse_et_al_2004a.pdf, acquired May 10, 2005.

Van House, N., Davis, M., Takhteyev, Y., Ames, M., \& Finn, M. (2004b) The Social Uses of Personal Photography: Methods for Projecting Future Imaging Applications. School of Information Management and Systems (SIMS), University of California, Berkeley, Working Papers, 2004. http:// www.sims.berkeley.edu/- vanhouse/vanhouseetal2004b.pdf, acquired May 13, 2005.

Van House, N., Davis, M., Ames, M., Finn, M. \& Viswanathan, V. (2005) 'The Uses of Personal Networked Digital Imaging: An Empirical Study of Cameraphone Photos and Sharing', in Extended Abstracts of the Conference on Human Factors in Computing Systems (CHI 2005) in Portland, Oregon, April 2-7, 2005. New York: ACM Press.

Weilenmann, A. (2003) Doing Mobility. Gothenburg: Göteborg University. (Gothenburg Studies in Informatics, Report 28).

Wiio, O. (1989) Viestinnän perusteet [The Principals of Communication]. Helsinki: Weilin + Göös.

Östman, J-O. \& Laakso, V. (1999) 'Postikortille diskurssia löytämässä' [Finding Discourse for the Postcard], in Laakso, Ville \& Östman, Jan-Ola (eds.) Postikortti diskurssina. Hämeenlinna: Korttien talo.

MIKKO VILLI, M.Soc.Sc. Researcher/doctoral student, School of Visual Culture/ Photography, University of Art and Design Helsinki, Hämeentie 135 C, FIN-00560 Helsinki, villi@uiah.fi 